\title{
Interactions between Kluyveromyces marxianus from cheese origin and the intestinal symbiont Bacteroides thetaiotaomicron: Impressive antioxidative effects
}

\author{
Alexandre Ceugniez, Mélissa Tourret, Elodie Dussert, Françoise Coucheney, \\ Barbara Deracinois, Philippe Jacques, Christophe Flahaut, Egon Heuson, Djamel Drider**, \\ Josette Behra-Miellet*
}

Univ. Lille, INRA, ISA, Univ. Artois, Univ. Littoral Côte d'Opale, EA 7394-ICV - Institut Charles Viollette, F-59000 Lille, France

\section{A R T I C L E I N F O}

\section{Article history:}

Received 12 September 2016

Received in revised form

23 February 2017

Accepted 30 March 2017

Available online 1 April 2017

\section{Keywords:}

Kluyveromyces marxianus

Bacteroides thetaiotaomicron

Anaerobic

Antioxidant

Probiotic

Chemical compounds studied in this article:

Acetonitrile (PubChem CID: 6342)

Acrylamide (PubChem CID: 6579)

$\alpha$-cyano-4-hydroxy-cinnamic acid

(PubChem CID: 5328791)

Ammonium bicarbonate (PubChem CID:

14013)

3-[(3-cholamidopropyl)

dimethylammonio]-1 propanesulfonate

(PubChem CID: 71774074)

Dithiotreitol (PubChem CID: 446094)

Formic acid (PubChem CID: 284)

Iodoacetamide (PubChem CID: 3727)

Ringer solution (PubChem CID: 56841909)

Trifluoroacetic acid (PubChem CID: 6422)

\begin{abstract}
A B S T R A C T
The effects of yeast Kluyveromyces marxianus S-2-05, of cheese origin, were assessed on the intestine anaerobe symbiont Bacteroides thetaiotaomicron ATCC 29741 to unveil any changes in its antioxidant properties. To this end, these microorganisms were grown and incubated either separately, or coincubated, under anaerobic atmosphere. Afterwards, the microbial cells were recovered and washed, and extracts were prepared using a sterile detergent solution to mimic the intestine detergent content. The extracts prepared from K. marxianus S-2-05 and reference strain K. marxianus MUCL 29917, grown under different conditions, were assessed for their antioxidant properties against superoxide anion and hydrogen peroxide. Extracts from both yeasts showed antioxidative effects, which were particularly important for K. marxianus S-02-5 after anaerobic incubation. Moreover, K. marxianus S-02-5 displayed a high level of activity against the aforementioned reactive oxygen species, enhancing that of B. thetaiotaomicron ATCC 29741, after the co-incubation process. Two-dimensional polyacrylamide gel electrophoresis was used to separate the proteins extracted. Superoxide dismutase, thiol peroxidase, rubrerythrin -intensively produced by $B$. thetaiotaomicron induced by the yeast-were identified by mass spectrometry. The antioxidative potential evidenced for $K$. marxianus S-02-5 is another advantage which could justify the utilization of this strain as a probiotic for countering intestinal inflammatory processes.
\end{abstract} ๑) 2017 Elsevier Ltd. All rights reserved.
Abbreviations: SOD, Superoxide dismutase; GR, glutathione reductase; ROS, reactive oxygen species; GYE, mediumglucose-yeast medium, aliquots of the extracts from both yeasts obtained after culture in broth ("K. marxianus S-02-5 liq" and "K. marxianus MUCL 29917 liq", respectively), aliquots of the extracts from both yeasts obtained after aerobic culture on agar medium ("K. marxianus S-02-5 surf" and "K. marxianus MUCL 29917 surf", respectively); TER, transepithelial electrical resistance; ACN, acetonitrile; DW, distilled water; TFA, Trifluoroacetic acid.

* Corresponding author.

** Corresponding author.

E-mail addresses: djamel.drider@univ-lille1.fr (D. Drider), josette.behra@univlille2.fr (J. Behra-Miellet).

\section{Introduction}

Bacteroides thetaiotaomicron can be considered as a model of anaerobic bacterium, a Gram-negative bacillus symbiont highly adaptable to its environment (Xu \& Gordon, 2003), and belonging to the predominant intestinal microbiota which is mainly composed of anaerobes. While this bacterium was well known before the 2000s as an opportunistic pathogen with an alarming profile of antibiotic resistance, a novel vision was then born after 
2004 indicating that $B$. thetaiotaomicron could have beneficial attributes for humans (Kelly et al., 2004). For the treatment of severe colitis or Crohn's disease, fecal microbiota transplantation is the infusion of liquid filtrate feces from a healthy donor (who is negative to a screening panel of laboratory exams) into the gut and B. thetaiotaomicron is a part of stool or fecal microbiota transferred to patients (Cammarota et al., 2015; Petrof \& Khoruts, 2014). This bacterium has also received Orphan Designation for paediatric Crohn's disease by the US Food and Drug Administration (2013). Previously, we established that under defined conditions, B. thetaiotaomicron displayed antioxidant and anti-inflammatory properties and antioxidant enzyme/proteins, chaperonin proteins produced by this bacterium were identified (Hochart-Behra, Drobecq, Tourret, Dubreuil, \& Behra-Miellet, 2014; Hochart-Behra et al., 2008). To gain more insights, we investigated the interactions between B. thetaiotaomicron ATCC 29741 and Kluyveromyces marxianus S-02-5 recently isolated from cheese and characterized for its beneficial attributes (Ceugniez, Drider, Jacques, \& Coucheney, 2015). Here we established that co-incubation of B. thetaiotaomicron ATCC 29741 with Kluyveromyces marxianus S02-5 could either retain or enhance its antioxidant potential. The antioxidative properties of yeasts were reported to induce microbial production of SOD (Dellomonaco et al., 2007). K. marxianus species were previously found to enhance superoxide dismutase (SOD) and glutathione reductase (GR) when they were exposed to hydrogen peroxide (Pinheiro, Belo, \& Mota, 2002), delineating their antioxidative properties. In direct line, Maccaferri et al. (Maccaferri, Klinder, Brigidi, Cavina, \& Costabile, 2012). reported the capacities of $K$. marxianus B0399 to diminish the levels of production of proinflammatory cytokines in PBMCs and Caco-2 cells, caused by stimulation with lipopolysaccharide.

The present work aimed at studying, under anaerobic conditions, the interactions between B. thetaiotaomicron ATCC 29741 and K. marxianus S-02-5. To this end, they were placed into a mild detergent mixture containing a cholic acid derivative preserving viability, in order to detect if these microorganisms are able to reject antioxidant elements from their surface at a higher level than previously described for $B$. thetaiotaomicron grown alone (HochartBehra et al., 2008, 2014). The activities against superoxide anion and hydrogen peroxide were measured in vitro. These reactive oxygen species (ROS) are the first described in the oxidative cascade of phagocytes (Hochart-Behra et al., 2008, 2014). Two-dimensional (2D) gels and matrix-assisted laser desorption ionization time of flight - Time of flight (MALDI TOF/TOF) were used to separate and identify any antioxidant enzymes/proteins in the compounds secreted both microorganisms.

\section{Materials and methods}

\subsection{Strains and growth conditions}

B. thetaiotaomicron strain ATCC 29741 (American Type Cultured Collection) and $K$. marxianus strain S-02-5 recently isolated from cheese (Ceugniez et al., 2015), stored at $-32{ }^{\circ} \mathrm{C}$, were revitalized and pre-cultured in/on suitable broth or agar medium before any experiment. B. thetaiotaomicron ATCC 29741 was grown on anaerobe broth (DIFCO, BD Dickinson, Le Pont de Claix, France), and yeasts K. marxianus strain S-02-5 and K. marxianus MUCL 29917, used as reference strain, were grown on GYE (glucose-yeast) agar medium ( $2 \%$ Glucose, $0.5 \%$ yeast extract, $1.5 \%$ Agar). Preliminary growth of $B$. thetaiotaomicron ATCC 29741 and yeasts were observed after a three-day incubation in anaerobic jars (AnaeroGen, Oxoid, Basingstoke, Hampshire, England), at $35-36{ }^{\circ} \mathrm{C}$ and aerobically at $30{ }^{\circ} \mathrm{C}$ for B. thetaiotaomicron ATCC 29741 and
K. marxianus S-02-5, respectively.

2.2. Interactions inter-species: B. thetaiotaomicron ATCC 29741 vs. K. marxianus $\mathrm{S}-02-5$

After a preliminary growth, B. thetaiotaomicron ATCC 29741 and K. marxianus S-02-5 were cultured separately or co-cultured anaerobically at $35-36{ }^{\circ} \mathrm{C}$ for two days in pre-reduced Brucella broth (DIFCO) supplemented with K3 vitamin (1 mg/L) (Sigma, Saint-Quentin Fallavier, France), suitable for Bacteroides. For K. marxianus S-02-5, the moistened yeast equivalent to $400 \mathrm{mg}$ of the dried microorganism (determined by drying the strains for 2.5 h using a SpeedVac) (LabConco Corporation, Kansas City, USA), was inoculated into the growth medium. Counts before and after B. thetaiotaomicron ATCC 29741 and K. marxianus S-02-5 together, were performed. After three-days co-culture of both strains together in a growth medium, the broth containing strains was transferred into pre-sterile centrifuge tubes, and tubes were centrifuged at $10,000 \mathrm{~g}$ for $13 \mathrm{~min}$ at $4{ }^{\circ} \mathrm{C}$. The supernatant was decanted off and the pellet cells were washed and diluted in Ringer $1 / 4$ medium (Oxoid, Hampshire, England). The spreading of the suitably diluted cells was done on Brucella agar surface (enriched with $\mathrm{K} 3$ vitamin and haemin at the concentrations of 1 and $5 \mathrm{mg} / \mathrm{L}$, respectively) to assess the viability and/or growth of microorganisms after co-culture. The same process was applied to B. thetaiotaomicron ATCC 29741 and K. marxianus S-02-5 grown concomitantly and separately, in the same conditions as for their co-culture. After centrifugation, followed by three successive washes in Ringer medium $1 / 4$, the pellets of pure B. thetaiotaomicron ATCC 29741 and of pure $K$. marxianus S-02-5 and of B. thetaiotaomicron ATCC 29741 co-culturing with K. marxianus S$02-5$ were placed in agitation in mild detergent solution containing $7 \mathrm{mM}$ 3-[(3-cholamidopropyl) dimethylammonio]-1 propanesulfonate, $0.5 \mathrm{mM}$ tris(hydroxymethyl) aminomethane hydrochloride at $\mathrm{pH} 6.8$ (Serva, Heidelberg, Germany) - a cholic acid derivative - overnight at $4{ }^{\circ} \mathrm{C}$, as previously described for B. thetaiotaomicron (Hochart-Behra et al., 2008, 2014) at a rate of $4 \mathrm{ml}$ of extraction solution per $\mathrm{g}$ of microorganisms. The suspensions were centrifuged and the supernatant was filtered $0.22 \mu$ (Millipore corporation, Bedford, MA, USA), and assayed for protein content (Hochart-Behra et al., 2008). The extracts were supposed to contain antioxidant compounds released from the surface of microorganisms. Counts were also performed before and after extraction in order to assess the viability of $B$. thetaiotaomicron ATCC 29741 and K. marxianus S-02-5. The in vitro pharmacological assays were then carried out as noted below.

\subsection{Comparison of intra yeast species: K. marxianus S-02-5 vs.} K. marxianus MUCL 29917

After the preliminary growth, both yeast strains $K$. marxianus S02-5 and K. marxianus MUCL 29917 were separately incubated in the pre-reduced Brucella broth as mentioned above, under anaerobic atmosphere, before proceeding with the extraction of elements secreted at the surface of the yeasts, using the method described above. The same extraction procedure was performed on both yeast strains collected from GYE agar after an aerobic two-day incubation.

The aliquots of the extracts from both yeasts were named “K. marxianus S-02-5 liq” and “K. marxianus MUCL 29917 liq” (after culture in broth), "K. marxianus S-02-5 surf" and "K. marxianus MUCL 29917 surf” (after aerobic culture on agar medium). Protein extracts were assayed using the Folin and Lowry method (Lowry, Rosebrough, Farr, \& Randall, 1951). 


\subsection{Pharmacological in vitro assays}

A superoxide anion produced using a cell-free model and hydrogen peroxide (Sigma) was placed into a reaction mixture at a concentration of about $10 \mathrm{nmol} / \mathrm{ml}$. Both ROS were in contact with increasing concentrations of the different extracts to study their inhibitory effect on ROS. Measurements of $\mathrm{O}_{2}^{-}$superoxide anion and $\mathrm{H}_{2} \mathrm{O}_{2}$ were made by spectrophotometry, according to Aruoma et al. (Aruoma, Halliwell, Hoey, \& Butler, 1989), and using methods largely tested and adapted to prevent interference (e.g., with peroxidases) (Leavey et al., 2002).

\subsection{Statistical analysis}

To study the variations between the populations of B. thetaiotaomicron ATCC 29741 and K. marxianus S-02-5 and K. marxianus MUCL 29917 during co-culture and extraction, the non-parametric sign-test was used at the $5 \%$ level ( $n=5$ independent experiments) and the Mann and Withney test was used at the $5 \%$ level, in case of small non-paired samples (Anastat). For pharmacological in vitro assays, the data was analysed from five independent assays using the Kruskal \& Wallis test at the 5\% level $(\mathrm{p}=0.05)$ (Anastat). Results were presented as box-plots where the extremities of the lowest and the highest bars represent the 10th and $90^{\text {th }}$ percentiles of percentages for each extract concentration. The 25 th, $50^{\text {th }}$ and 75 th percentiles correspond to the inferior, interior and superior horizontal bars of the boxes constructed for each extract concentration.

\subsection{Proteomic analysis}

The extracts (all at a volume equivalent to $500 \mu \mathrm{g}$ proteins secreted by $K$. marxianus S-02-5) were desalted, re-solubilized, lyophilized and the protein separation performed in gels as previously described for 2D PAGE (Hochart-Behra et al., 2014). Proteins were loaded on precast immobilized $\mathrm{pH}$ 3-10 gradient ReadyStrip (17 cm, BioRad) and isoelectric focusing (IEF) was performed in the BioRad Protean IEF cell system. Vertical gels with 4-20\% acrylamide (BioRad) gradient (non-denaturing gels) were performed for SDS PAGE. Separate proteins in the gels were then stained (colloidal Coomassie blue) fixed and washed. Gel images were acquired using a GS800 densitometer (BioRad). PDQuest software (BioRad) was used to analyze the protein profile of the 2D-gels of $\mathrm{pH} \mathrm{3-10}$ gradient. Protein spots of interest were excised and prepared to perform protein identification by peptide mass finger printing technique using MALDI TOF/TOF mass spectrometer. They were ingel trypsin-digested according a method adapted from that of Shevchenko et al.(Shevchenko, Wilm, Vorm, \& Mann, 1996). Protein spots were first discolored [20 mM ammonium bicarbonate (Prolabo, Fontenay-Sous-Bois, France), 50\% (vol/vol) acetonitrile (ACN, Carlo Erba Reagents) in distilled water (DW) solution], dried by rotary evaporation (miVac QUATTRO concentrator, Genevac ${ }^{\circledR}$, Ipswich, England). Then reduction for $45 \mathrm{~min}$ at $56{ }^{\circ} \mathrm{C}[20 \mathrm{mM}$ ammonium bicarbonate, $10 \mathrm{mM}$ 1,4-dithiotreitol in DW solution (Sigma)], removal of supernatants and alkylation for $45 \mathrm{~min}$ at $20{ }^{\circ} \mathrm{C}$ in the dark [20 mM ammonium bicarbonate, $55 \mathrm{mM}$ iodoacetamide (Sigma)] were performed. After removal of supernatants, washing of the gels with acetonitrile and drying by rotary evaporation, the trypsin digest was then made using a $12,5 \mathrm{mg} \mathrm{L}^{-1}$ trypsin solution (Trypsin/Lys-C Mix, Promega, Madison, WI). The peptides were extracted from protein spots with $50 \%$ ACN, $50 \%$ ammonium bicarbonate in DW ( $\mathrm{vol} / \mathrm{vol}$ ) solution and finally with a solution of $0.5 \%$ formic acid in ACN. The supernatants were pooled before being concentrated and desalted on a ZipTip $\mu-\mathrm{C} 18^{\mathbb{B}}{ }^{\text {column (Mil- }}$ lipore). The peptides were eluted by an $80 \% \mathrm{ACN}, 0.1 \%$ trifluoroacetic acid (TFA) (vol/vol) solution. Mass analysis was performed with a Bruker Autoflex Speed MALDI TOF/TOF mass spectrometer (Bruker-Franzen Analytik, Bremen, Germany) according Shevchenko et al.(Shevchenko et al., 1996). The MALDI target plate (AnchorChip ${ }^{\mathrm{TM}}$ ) was covered with extracted peptides mixed with $\alpha$-cyano-4-hydroxy-cinnamic acid matrix $(2.8 \mathrm{mg} / \mathrm{ml}$ in ACN:TFA, 85:0.1 vol/vol). The molecular mass measurements were performed in automatic mode using FlexControl ${ }^{\mathrm{TM}} 3.4$ software on an Autoflex Speed ${ }^{\mathrm{TM}}$ TOF/TOF instrument and in reflectron mode for MALDI-TOF peptide mass fingerprinting (PMF) or LIFT mode for MALDI-TOF/TOF peptide fragmentation fingerprinting (PFF). External calibration over the 1000-3500 mass range was performed using the $[\mathrm{M}+\mathrm{H}]+$ monoisotopic ions of bradykinin 1-7, angiotensin I, angiotensin II, substance $\mathrm{P}$, bombesin and adrenocorticotropic hormone (clips 1-17 and clips 18-39) from a peptide calibration standard kit (Bruker Daltonik). Each spectrum was produced by accumulating data from 1200 laser shots. A maximum of ten precursor ions per sample were chosen for LIFT-TOF/TOF MS/ MS analysis. Peak lists were generated from MS and MS/MS spectra using Flexanalysis ${ }^{\mathrm{TM}} 3.4$ software (Bruker Daltonik). Database searches using combined PMF and/or PFF datasets were performed in the UniProt and NCBInr databases via BioTools 3.2 (Bruker Daltonik) [with Mascot 2.2 (Matrix Science Ltd, London, UK)] or PEAKS Studio 7.0 (Bioinformatics Solutions) or Profound (taxonomy restricted to Bacteria, Yeast or not restricted). A mass tolerance of $150 \mathrm{ppm}$ and 1 missing cleavage site for PMF and an MS/MS tolerance of 0.5 Da and 1 missing cleavage site for MS/MS searching were allowed. Variable cysteine carbamidomethylation and methionine oxidation were also considered. The relevance of protein identities was judged according to their score in the research software ( $p$ value of 0.05 ( $p<0.05$ ), False Discovery Rate $<1 \%$ ). Searching was also made with Mascot in the National Center for Biotechnology Information (NCBI), SwissProt and Uniprot databases.

\section{Results}

\subsection{The interaction benefited the intestine bacteria and the yeast population was stable}

As expected, when grown alone under anaerobic conditions, the cell count of $B$. thetaiotaomicron ATCC 29741 increased by about 1 $\log$ (CFU/L of culture) $(p=0.0313)$. On the other hand, the yeast cell count remained stable during transit in the anaerobic atmosphere. Means of CFU counts varied from $10.35 \pm 0.07 \log$ to $10.22 \pm 0.09$ log. Nevertheless, during co-incubation, the cell count of B. thetaiotaomicron ATCC 29741, expressed in CFU/L of culture, significantly increased by a mean value of about $0.9 \log (p=0.0313)$ whilst that of K. marxianus S-02-5 has a drop of about $0.3 \log$ CFU/L of culture. Clearly, B. thetaiotaomicron ATCC 29741 took advantage of this interaction but the cell counts of yeasts remained stable. During the extraction of the surface elements from these microorganisms, the viabilities of $K$. marxianus $\mathrm{S}-02-5$ and B. thetaiotaomicron ATCC 29741 were also stable after single cultures. The counts of these populations, expressed in log CFU per total suspension of microorganisms in solution of extraction, were $10.33 \pm 0.27 \log$ before extraction and $11.07 \pm 0.27 \log$ after extraction $(p=0.6875)$ for $B$. thetaiotaomicron ATCC 29741; $9.93 \pm 0.45 \mathrm{log}$ before extraction and $9.81 \pm 0.56 \mathrm{log}$ after extraction for $K$. marxianus S-02-5 ( $p=0.1875)$. The bacterial population remained stable overall during the extraction after co-culture. Indeed, the counts ranged from $10.71 \pm 0.81$ to $10.96 \pm 1.17 \mathrm{log}$, with $p$ value 0.500 , and a small but not statistically significant loss was observed for $K$. marxianus S-02-5, the counts of which ranged from $10.08 \pm 0.08$ to $9.88 \pm 0.63 \log$ with $p$ value of 0.1875 . Notably, 
similar protein quantities were found in the extracts $(2.94 \pm 1.75$, $1.82 \pm 0.94$ and $1.36 \pm 0.42 \mathrm{mg} / \mathrm{ml}$ in the extracts related to coculture, to ATCC 29741 grown alone and to K. marxianus S-02-5, respectively).

As depicted in Figs. 1 and 2, the data of the pharmacological in vitro assays show the effects of the extracts on superoxide anion and hydrogen peroxide, respectively. The antioxidative effect of the extract from the co-culture appeared to be similar against superoxide anion (concentration of inhibition at $50 \%$ or IC50 of about $51 \mu \mathrm{l}$ of extract $/ \mathrm{ml}$ ) and higher against hydrogen peroxide (IC50 of about $31 \mu \mathrm{l}$ of extract.ml), than the effect of the extract obtained from B. thetaiotaomicron ATCC 29741 alone. The IC50 was $51 \mu \mathrm{l}$ of extract $/ \mathrm{ml}$ for superoxide anion, and was not reached at the highest concentration of extract tested on hydrogen peroxide. The extract obtained from $K$. marxianus S-02-5 cultured alone inhibited superoxide anion at the concentration of $10 \mathrm{nmol} / \mathrm{ml}$ with an IC50 of about $25 \mu \mathrm{l}$ of extract/ml. IC50 for hydrogen peroxide was obtained at $6 \mu \mathrm{l}$ of extract $/ \mathrm{ml}$.

The protein analysis of the $B$. thetaiotaomicron extract revealed the presence of antioxidant or oxidation-reduction enzymes (2D gel and protein identifications available in Supplementary material in Fig. S1 and Tables S5 and S6) such as thiol peroxidase (spots 01 and 02 ), ferritin (03), antibiotic biosynthesis monooxygenase (04). However, superoxide dismutase is not detected in these extracts. As previously described (Hochart-Behra et al., 2008), chaperonin proteins were also found such as chaperone protein DnaK (c1), SCO1/SenC chaperone. Glutamate decarboxylase (s1), is another stress protein previously widely described in our extracts (HochartBehra et al., 2008). The other proteins concern the carbohydrate catabolic pathway from Bacteroides (phosphoenolpyruvate carboxykinase in spots $\mathrm{p} 1$ and $\mathrm{p} 2$, lactate/malate dehydrogenase in spots p5 and p6, glyceraldehyde-3-phosphate dehydrogenase in spots p7, p9 to p11). Tetratricopeptide repeat protein (spot p3), magnesium chelatase, subunit I/ATPase (p4) and glutaminase (in

\section{Superoxide anion $(\mathrm{nmol} / \mathrm{mL})$}

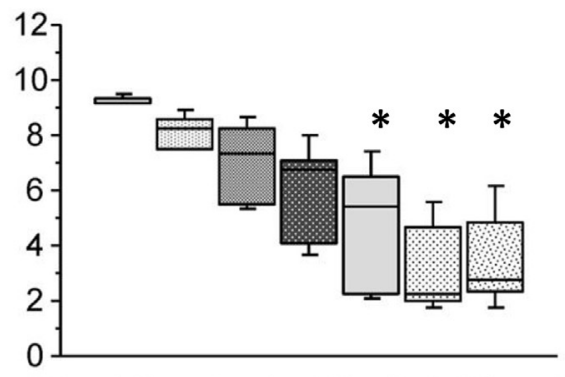

Extract from Bacteroides thetaiotaomicron
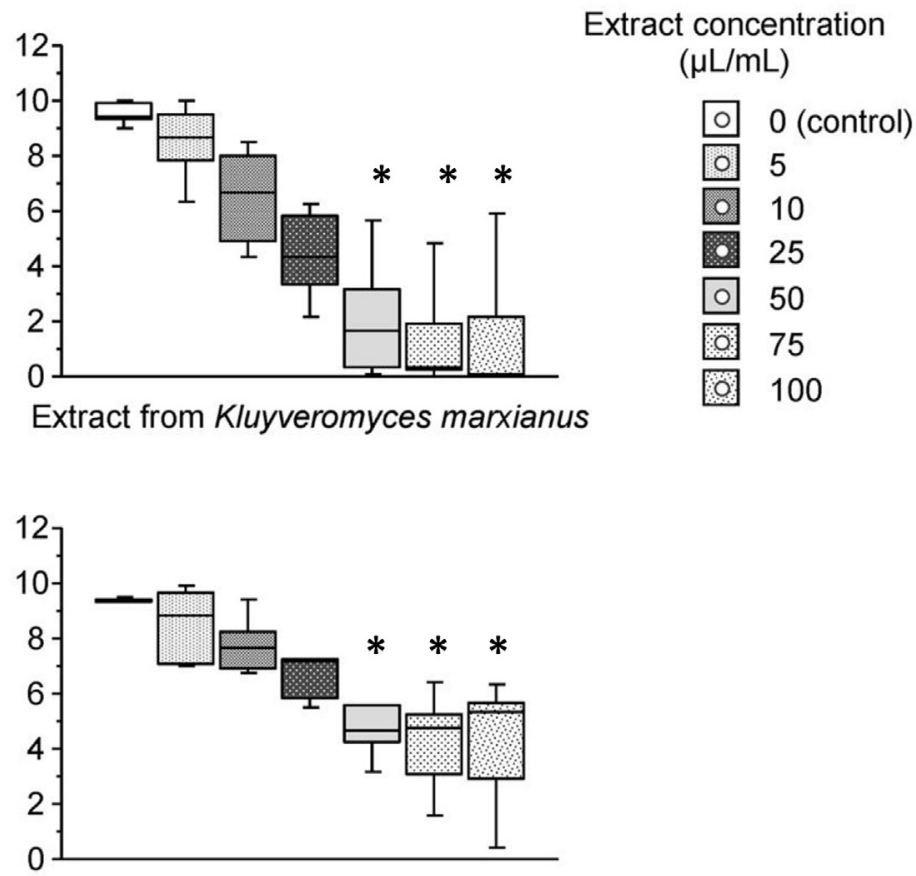

Extract from the $B$. thetaiotaomicron/K. marxianus co-culture

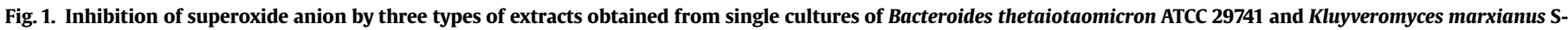

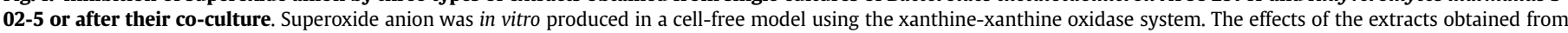

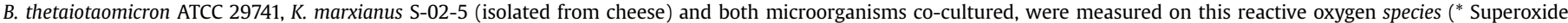

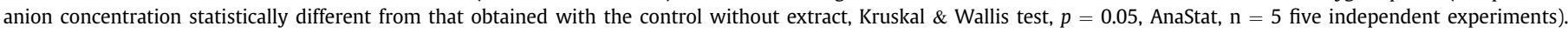
Means \pm standard deviations are available in Supplementary Material Table S1. 


\section{Hydrogen peroxide}

$(\mathrm{nmol} / \mathrm{mL})$

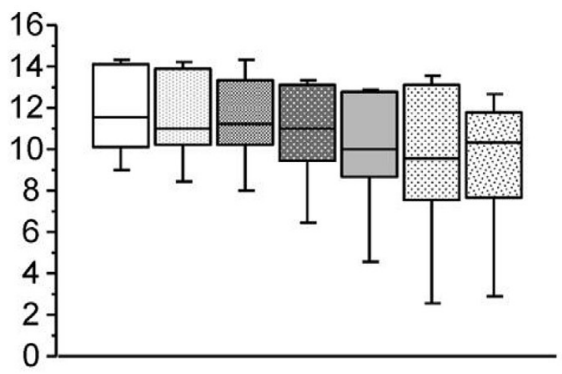

Extract from Bacteroides thetaiotaomicron
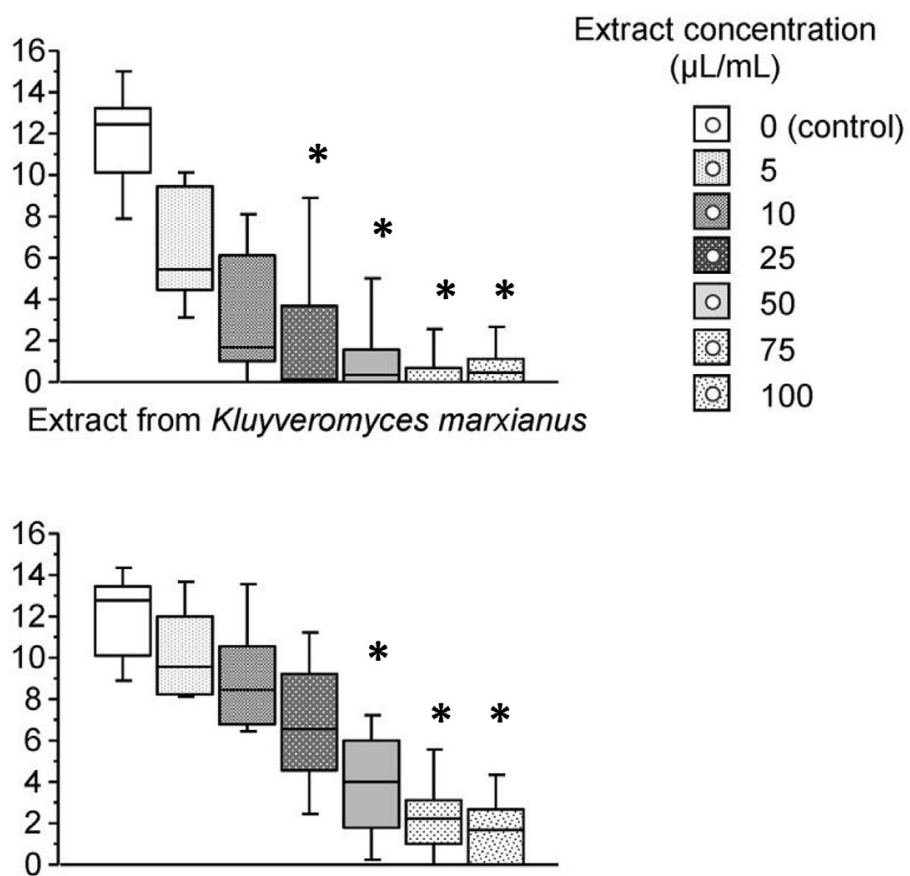

Extract from the $B$. thetaiotaomicron/K. marxianus co-culture

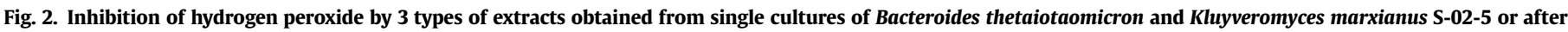

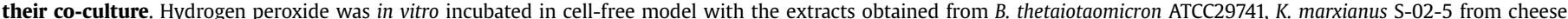

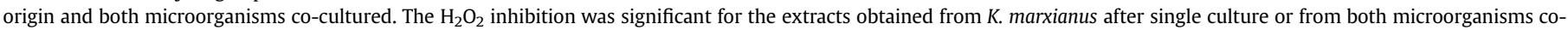

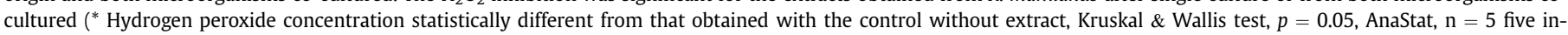
dependent experiments). Means \pm standard deviations are available Supplementary Material Table S2.

spot p8) were also found.

As native 2D-gels were used for protein migration, protein associations could be possible such as dimers (or more associated monomers) and they could migrate at different molecular weights in the gels. With this technique, protein interactions can be visualized and one spot can correspond to several proteins. Conversely, although our method was less denaturing for the proteins migrating in the gels, their breaking in fragments could however occur and appear at a molecular weight lower than expected.

The proteins extracted from both co-cultivated microorganisms were mainly identified for B. thetaiotaomicron (Fig. 3; protein identification available in Supplementary Material Tables S7 and S8) in different categories: the group of antioxidant proteins with superoxide dismutase (spots BT-o1and BT-o2), rubrerythrin (BTo3), probable thiol peroxidase/lipid hydroperoxide peroxidase (BTo4 to BT-07), the group of chaperones with the Chaperone protein DnaK (BT-c1), Molecular chaperone DnaJ (BT-c2), Cationic outer membrane protein OmpH (BT-c3), Heat shock protein Hsp20 (BTc4), $10 \mathrm{kDa}$ chaperonin (BT-c5) and other stress proteins such as Glutamate decarboxylase (BT-s1 and BT-s2). Proteins of the carbohydrate catabolic pathway from Bacteroides were also found in these extracts (phosphoenolpyruvate carboxykinase in spots BT-p1, BT-p3 and BT-p4 or lactate/malate dehydrogenase/glyceraldehyde3-phosphate dehydrogenase (in spots BT-p2 and BT-p6 to BT-p9) or 2,3-bisphosphoglycerate-dependent phosphoglycerate mutase in spots BT-p12 and BT-p13. Succinate-CoA ligase in spot BT-p14), NAD dependent epimerase (BT-p15) were also identified. Enolase was found for K. marxianus S-02-5 (K-p1). Tetratricopeptide repeat protein (BT-p5), Choloylglycine hydrolase (BT-p10), Glutaminase (BT-p11).

In the extract obtained from $K$. marxianus S-02-5, protein analysis (2D gel and protein identifications available in Supplementary Material Fig. S2 and Tables S9 and S10) could underpin among others the presence of glucose-6-phosphate isomerase (p3), 


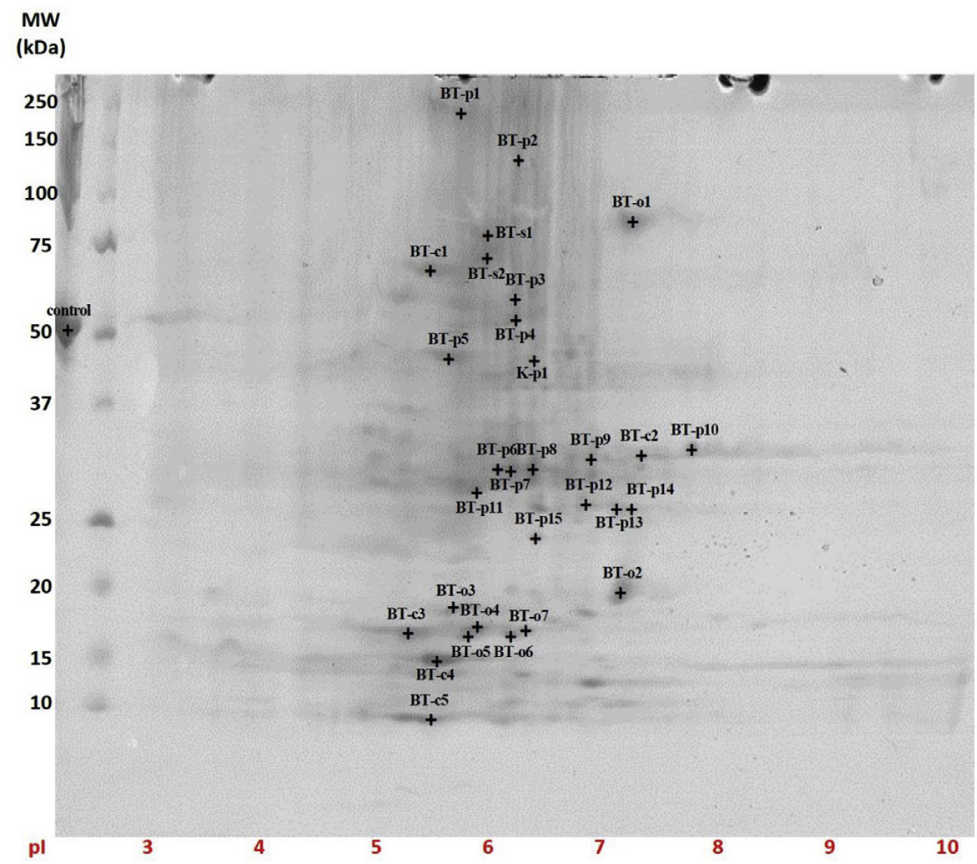

\begin{tabular}{|c|c|c|}
\hline & Identified protein & $\begin{array}{c}\text { Numbering } \\
\text { in gel }\end{array}$ \\
\hline \multirow{7}{*}{$\begin{array}{l}\text { Oxidative } \\
\text { stress } \\
\text { proteins }\end{array}$} & Superoxide dismutase ( $B$, thetaiotaomicron) & BT-o1 \\
\hline & Superoxide dismutase (B. thetaiotaomicron) & BT-02 \\
\hline & Rubrerythrin (B. thetaiotaomicron) & BT-03 \\
\hline & Thiol peroxidase (B. thetaiotaomicron) & BT-o4 \\
\hline & Thiol peroxidase (B. thetaiotaomicron) & BT-05 \\
\hline & Thiol peroxidase (B. thetaiotaomicron) & BT-o6 \\
\hline & Thiol peroxidase (B. thetaiotaomicron) & BT-07 \\
\hline \multirow{5}{*}{$\begin{array}{l}\text { Chaperonin } \\
\text { proteins }\end{array}$} & Chaperone protein DnaK (B. thetaiotaomicron) & BT-cl \\
\hline & Chaperone protein DnaJ (B. thetaiotaomicron) & BT-c2 \\
\hline & Outer membrane protein $\mathrm{OmpH}$ (B. thetaiotaomicron) & BT-c3 \\
\hline & Heat shock protein $\mathrm{Hsp} 20$ (B. thetaiotaomicron) & BT-c4 \\
\hline & $10 \mathrm{kDa}$ chaperonin (B. thetaiofaomicron) & $\mathrm{BT}-\mathrm{c} 5$ \\
\hline \multirow{2}{*}{$\begin{array}{l}\text { Other } \\
\text { stress } \\
\text { proteins }\end{array}$} & Glutamate decarboxylase (B. thetaiotaomicron) & BT-s1 \\
\hline & Glutamate decarboxylase (B. thetaiotaomicron) & BT-s2 \\
\hline \multirow{16}{*}{$\begin{array}{l}\text { Other } \\
\text { proteins }\end{array}$} & Phosphoenolpyruvate carboxykinase (ATP) (B. thetaiotaomicron) & BT-p1 \\
\hline & Malate dehydrogenase (B. thetaiotaomicron) & BT-p2 \\
\hline & Phosphoenolpyruvate carboxykinase (ATP) (B. thetaiotaomicron) & BT-p3 \\
\hline & Phosphocnolpyruvate carboxykinase (ATP) (B. thetaiotaomicron) & BT-p4 \\
\hline & Tetratricopeptide repeat protein (B. thetaiotaomicron) & BT-p5 \\
\hline & Lactate/malate dehydrogenase (B. thetaiotaomicron) & BT-p6 \\
\hline & Lactate/malate dehydrogenase (B. thetaiotaomicron) & BT-p? \\
\hline & $\begin{array}{l}\text { Lactate/malate dehydrogenase or glyceraldehyde-3-phosphate } \\
\text { dehydrogenase (B. thetaiotaomicron) }\end{array}$ & BT-p8 \\
\hline & Glyceraldehyde-3-phosphate dehydrogenase (B. thetaiotaomicron) & BT-p9 \\
\hline & Choloylglycine hydrolase (B. thetaiotaomicron) & BT-p10 \\
\hline & Glutaminase (B. thetaiotaomicron) & BT-p11 \\
\hline & $\begin{array}{l}\text { 2,3-biphosphoglycerate-dependent phosphoglycerate mutase ( } B \text {. } \\
\text { thetaiotaomicron) }\end{array}$ & BT-p12 \\
\hline & $\begin{array}{l}\text { 2,3-biphosphoglycerate-dependent phosphoglycerate mutase ( } B \text {. } \\
\text { thetaiotaomicron) }\end{array}$ & BT-p13 \\
\hline & Succinate-CoA ligase (ATP-forming) (B. thetaiotaomicron) & BT-p14 \\
\hline & NAD dependent epimerase/dehydratase ( $B$. thetaiotaomicron) & BT-p15 \\
\hline & Enolase ( $K$. marxiamus) & K-p1 \\
\hline Control & Serum albumin (Bos taurus) & Control \\
\hline
\end{tabular}

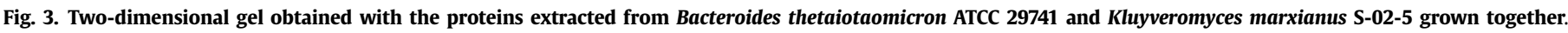

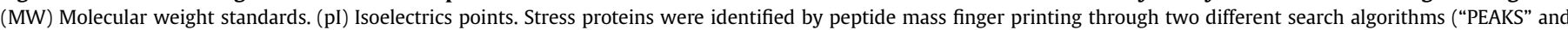

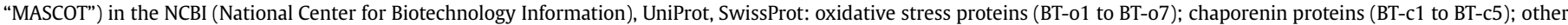
stress proteins (BT-s1, BT-s2); other proteins (BT-p1 to BT-p15; K-p1); control (serum albumin).

hexokinase (in spot p4), enolase (p1, p5, p7, p12, p14, p19, p20), enoate reductase 1 (p10), phosphoglycerate kinase ( $\mathrm{p} 9, \mathrm{p} 12, \mathrm{p} 13$, $\mathrm{p} 15),($ exo)inulinase ( $\mathrm{p} 2), \mathrm{NAD}(\mathrm{P}) \mathrm{H}$-dependent $\mathrm{D}$-xylose reductase or Xyl1 (o2), NADPH-dependent alpha-keto amide reductase (01), phosphoglycerate mutase 1 (p16 à p18), peptidyl-prolyl cis-trans isomerase ( $\mathrm{s} 1)$.

\subsection{Comparison of both K. marxianus strains: stability of the yeasts incubated under anaerobic condition or during the extraction of antioxidant metabolites}

K. marxianus S-02-5 and K. marxianus MUCL 29917 strains were compared for their antioxidant potential. Thus, their incubation under anaerobic conditions at $35-36{ }^{\circ} \mathrm{C}$ confirmed the stability of the cell counts of K. marxianus S-02-5 or K. marxianus MUCL 29917 under these conditions. Remarkably, both yeasts survived during the process of extraction as indicated by colony forming unit (CFU) counts (only $0.1 \log$ CFU-loss during the extraction). When yeasts were collected at the GYE surface after aerobic culture, cell counts before and after extraction underlined the stability of $K$. marxianus S-02-5 and K. marxianus MUCL 29917 during the extraction $(p>0.05)$.

Overall, the protein contents of the extracts appeared to depend on the method of yeast production. The mean protein concentration of the extract " $K$. marxianus S-02-5 liq" $(2.6 \pm 0.41 \mathrm{mg} / \mathrm{ml})$ was tenfold higher than that of " $K$. marxianus $S-02-5$ surf" $(0.25 \pm 0.14 \mathrm{mg} / \mathrm{ml})$. For “K. marxianus MUCL 29917 liq”, protein concentration was quadrupled $(2.08 \pm 0.35 \mathrm{mg} / \mathrm{ml})$, relatively to that of "K. marxianus MUCL 29917 surf" $(0.53 \pm 0.31 \mathrm{mg} / \mathrm{ml})$.

After growth of $K$. marxianus S-02-5 and $K$. marxianus MUCL 29917 under anaerobic conditions in broth, the mean $\mathrm{IC}_{50}$ of the extracts against superoxide anion were about $7 \mu \mathrm{l} / \mathrm{ml}$ for both “K. marxianus S-02-5 liq" and "K. marxianus MUCL 29917 liq" (Fig. 4). A strong action of both extracts was found against hydrogen peroxide, with however a superiority to "K. marxianus S-02-5 liq" which IC50 was still of about $5 \mu$ of extract/ml. That of " $K$. marxianus MUCL 29917 liq” reached $14 \mu \mathrm{l}$ of extract/ml (Fig. 5).

After aerobic growth of yeast strains on GYE agar, an inhibitory effect was observed for the extract "K. marxianus S-02-5 surf" against superoxide anion, with a maximal activity (39.6\% inhibition of the positive control of $\mathrm{O}_{2}^{-}$production) at $100 \mu \mathrm{l}$ of extract/ml. For "K. marxianus MUCL 29917 surf" the maximal mean inhibition was higher with $84.6 \%$ at $100 \mu \mathrm{l}$ of extract/ml (IC $5030.75 \mu \mathrm{l}$ of extract/ $\mathrm{ml}$ ). The maximal mean inhibitions of hydrogen peroxide of $23.5 \%$ and $43 \%$ were observed for " $K$. marxianus S-02-5 surf" and " $K$. marxianus MUCL 29917 surf" at $100 \mu \mathrm{l}$ of both extracts/ml, respectively.

\section{Discussion}

In the intestinal environment, the interactions between the symbiont $B$. thetaiotaomicron, known for its anti-inflammatory and antioxidative properties (Hochart-Behra et al., 2008, 2014) and K. marxianus, were reported to produce antioxidant enzymes. Here, novel insights regarding the anti-oxidative properties of the anaerobe in contact with yeast isolated from cheese K. marxianus S02-5 and K. marxianus MUCL 29917 used as control were examined and the cell counts of these microorganisms were determined. Overall, the experiments performed here were aimed at establishing whether $K$. marxianus S-02-5, recently isolated from cheese, could provide further probiotic attributes for its utilization in inflammatory bowel diseases.

The interactions between B. thetaiotaomicron ATCC 29741 and K. marxianus S-02-5 and K. marxianus MUCL 29917 privileged the B. thetaiotaomicron ATCC 29741 population under anaerobic conditions. The extracts obtained in the detergent solution, mimicking therefore the intestinal detergent conditions, confirmed an inhibitory effect of $B$. thetaiotaomicron ATCC 29741 metabolites on both $\mathrm{O}_{2}^{-}$and $\mathrm{H}_{2} \mathrm{O}_{2}$. The proteins identified in the intense spots in the gel, relative to the bacterial extract, were similar to those previously 
Superoxide anion

$(\mathrm{nmol} / \mathrm{mL})$

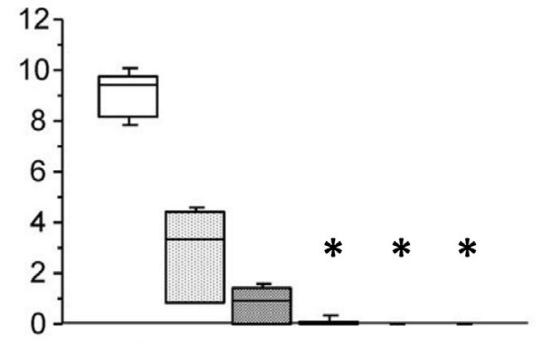

Extract from Kluyveromyces marxianus S-02-5 liq
Extract concentration

$(\mu \mathrm{L} / \mathrm{mL})$

0 (control

- 10

o 25

20 50

(0) 75

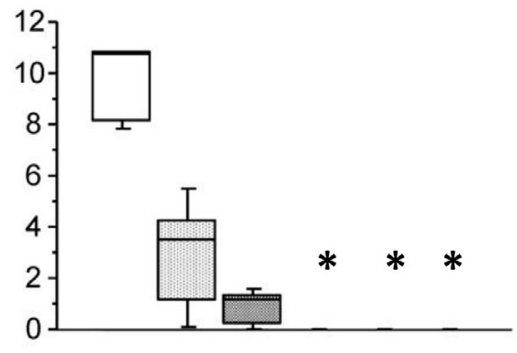

요 100

Extract from Kluyveromyces marxianus MUCL $29917 \mathrm{liq}$

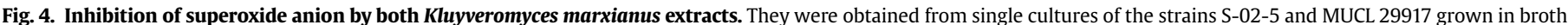

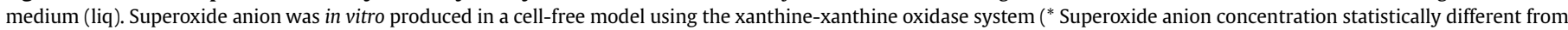

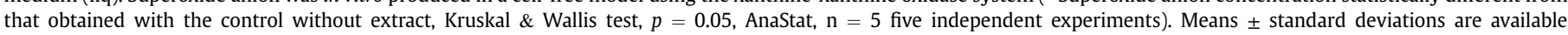
Supplementary Material Table S3.

\section{Hydrogen peroxide $(\mathrm{nmol} / \mathrm{mL})$}

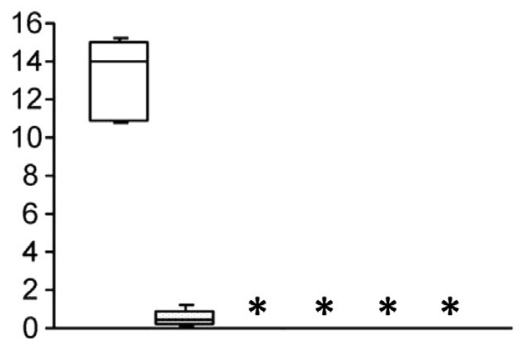

Extract from Kluyveromyces marxianus S-02-5 liq

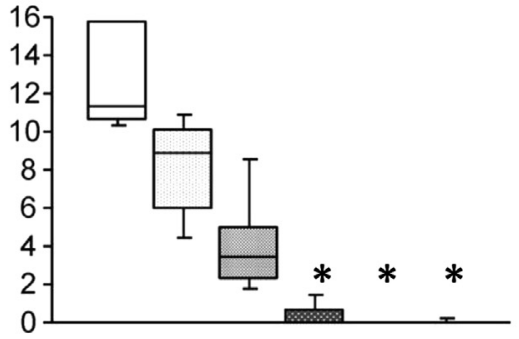

\section{Extract concentration} $(\mu \mathrm{L} / \mathrm{mL})$

0 (control)

o 10

25

- 50

ㄱ. 75

Extract from Kluyveromyces marxianus MUCL 29917 liq

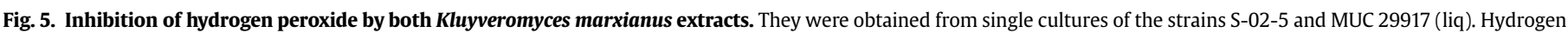

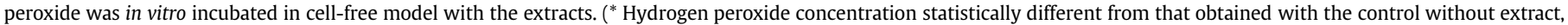
Kruskal \& Wallis test, $\mathrm{p}=0.05$, AnaStat, $\mathrm{n}=5$ five independent experiments). Means \pm standard deviations are available Supplementary Material Table S4.

reported (Hochart-Behra et al., 2014), such as ferritin, thiol peroxidase. They are likely involved in the antioxidative effects observed for the extract. However, SOD was not detected, especially in its hexameric form (Hochart-Behra et al., 2008). Stress proteins and 
chaperones were also found as expected in this type of extract: DnaK, SCO1/SenC chaperone which family has been extensively studied for its role in the incorporation of copper in the catalytic site of oxidases (Fujimoto et al., 2012) and other proteins such as glutamate decarboxylase (largely described in our previous extracts) which genes are known to be positively regulated by acidic shock, salt stress and stationary growth phase in Escherichia coli (De Biase, Tramonti, Bossa, \& Visca, 1999). Magnesium chelatase, subunit I/ATPase was identified in the $B$. thetaiotaomicron extract whose ATPase activity could be regulated by thioredoxin redox. In pea plants, it has been reported to modulate the redox-mediated signaling in tetrapyrrole biosynthesis and the homeostasis of reactive oxygen species (Luo et al., 2012). A group of main enzymes involved in the carbohydrate catabolic pathway of Bacteroides which lead to acetate, butyrate, propionate, succinate and lactate (Fischbach \& Sonnenburg, 2011) was found here yet. We identified a tetratricopeptide repeat protein (TPR). It could participate in the formation of protein complexes. TPRs generally form amphipathic helix pairs that mediate protein-protein interactions and multiprotein complex assemblies with other TPR-containing or nonTPR-containing proteins (Bakolitsa et al., 2010). Such patterns have been described for a protein complex of $B$. thetaiotaomicron involved in the starch utilization. Under our experimental conditions, we had previously shown using electronic microscopy that the anaerobic bacterium exported to the extraction medium an assembly of survival proteins in the form of budding vesicles which were probably responsible for the antioxidant effect of the extract (Hochart-Behra et al., 2014).

The effect of the extract on ROS was enhanced especially on $\mathrm{H}_{2} \mathrm{O}_{2}$, when both microorganisms (K. marxianus and $B$. thetaiotaomicron) were co-incubated. Moreover, the viabilities of both microorganisms were stable during the extraction. The effect of the extract obtained from $K$. marxianus S-02-5 incubated alone in broth under anaerobic conditions showed strong antioxidant activity. Indeed, as little as $25 \mu \mathrm{l}$ of extract $/ \mathrm{ml}$ was sufficient to inhibit $50 \%$ of superoxide anion or hydrogen peroxide. The superoxide dismutase from $K$. marxianus was previously reported to act efficiently against $\mathrm{O}_{2}^{-}$(Stefanova, Ratcheva, Nikolova, \& Neychev, 2014), and could explain the inhibition observed here upon addition of our extracts. Curiously, no yeast SOD was identified among the major spots in the gels relative to the extracts resulting from the co-culture or from $K$. marxianus S-02-5 grown alone. The eukaryotic yeast $\mathrm{Cu} / \mathrm{Zn}$ SOD has been studied and would appear in gel at around $34 \mathrm{kDa}$ as dimeric form or at $17 \mathrm{kDa}$ as monomer (Dellomonaco et al., 2007; Nedeva et al., 2009; Stefanova et al., 2014). K. marxianus was viable during the extraction arguing that cell disruption did not occur during the process. The K. marxianus SOD is cytosolic and was not released into the extraction medium. Furthermore, we could also expect the presence of peroxidases and/or of thiol-containing proteins in the extracts, countering $\mathrm{H}_{2} \mathrm{O}_{2}$. The unique yeast protein identified in 2D-gel performed with the extract from the co-culture was enolase, enzyme of the glycolytic pathway. The yeast seemed overshadowed by the contact of $B$. thetaiotaomicron after anaerobic co-culture. As a reminder, $B$. thetaiotaomicron is able to produce a large amount of acetate via the carbohydrate anaerobic pathway. It has been recently reported (Martynova et al., 2016) that K. marxianus was inhibited in its growth by acetate. It could extend lag phase, during which cells adapt to elevated acetate concentrations, and growth reoccurs at a slower rate. In contrast, the anaerobe seemed rapidly benefit from the presence of yeast and produced key-antioxidant/stress proteins, much more abundant way than when it is cultivated at the same level out of the presence of the yeast. The full range of enzymes/proteins useful in the detoxification of ROS was highlighted in 2D-gels, especially the SOD in its hexameric (in spot 01 in Fig. 3) or monomeric (spot BT-o2 in Fig. 3) form. The presence in gels of an intense spot of SOD hexamer seemed related to a powerful effect against superoxide anion (Hochart-Behra et al., 2008). Rubrerythrin (spot BT-03), probable thiol peroxidase/lipid hydroperoxide peroxidase (spots BT-04 to BT-o7) as shown in Fig. 3 constitute a full arsenal against $\mathrm{H}_{2} \mathrm{O}_{2}$. Rubrerythrins are non-heme di-iron proteins involved in oxidative stress defense as peroxide scavengers in a wide range of organisms. These proteins belonging to the ferritinlike superfamily contain a C-terminal rubredoxin-like domain involved in electron transport used during catalysis in anaerobic conditions (Cardenas, Quatrini, \& Holmes, 2016). B. thetaiotaomicron, was reported by Mishra and Imlay (Mishra \& Imlay, 2013) to use a consortium of enzymes to scavenge hydrogen peroxide, and rubrerythrin devoid of any secretory leader sequence. It appears, for these authors, to be devoted to scavenging $\mathrm{H}_{2} \mathrm{O}_{2}$ in the cytoplasmic compartment. However, in our study, the bacterial cell viability was preserved and we observed an intense protein spot for rubrerythrin in 2D-gel. Our previous studies have also shown a particular protein export mode by budding of vesicles on the bacterial wall which could be used for rubrerythrin and peroxidase export. Chaperones are useful for refolding aggregated proteins after stress such as (in Fig. 3) DnaK (in spot BT-c1), DnaJ (BT-c2), cationic outer membrane protein OmpH (BT-c3), Heat shock protein Hsp20 (BT-c4), $10 \mathrm{kDa}$ chaperonin (BT-c4) and other stress proteins such as glutamate decarboxylase (BT-s1 and BT-s2). So the $B$. thetaiotaomicron glutaminase found in our extract may be essential to provide from glutamine the glutamate substrate to glutamate decarboxylase. TPR was once again identified in the extract, performed this time with both microorganisms. The extract from the co-culture contained also the full enzyme necessary for the carbohydrate catabolism et to provide energy (Miller \& Wolin, 1979). As for choloylglycine hydrolase, this enzyme catalyzes the hydrolysis of amide bonds of conjugated bile salts and could act under our experimental conditions on the 3-[(3-cholamidopropyl) dimethylammonio]-1 propanesulfonate detergent mimicking bile salts (Ridlon, Kang, \& Hylemon, 2006).

When the extract was made with $K$. marxianus S-02-5 only, the identification of the protein in the gel has especially shown glycolysis enzymes, (exo)inulinase, Xyl1 which is, NADPHdependent alpha-keto amide reductase. (Exo)inulinase from $K$. marxianus is a powerful extracellular enzyme of pharmaceutical interest, which rapidly hydrolyzes inulin to produce fructooligosaccharides and fructose whose metabolism bypasses the metabolic pathway of glucose and therefore does not require insulin (Singh \& Lotey, 2010). Inulinase from K. marxianus exists in two forms. The inulinase present in the culture fluid is a dimer, and the enzyme retained in the cell wall is a tetramer. In our case, the spot p2 found at a molecular weight of around $125 \mathrm{kDa}$ in the nondenaturing gel (available in supplementary content 7) could be compatible with the dimer (Rouwenhorst, Hensing, Verbakel, Scheffers, \& van Duken, 1990). K. marxianus is capable of growing under anaerobic fermentation conditions that lead to ethanol production even if alternating aerobic and anaerobic stages has been shown to promote the growth of the yeast. K. marxianus is also capable to use various hexoses or pentoses for its fermentation to ethanol (Banat, Singh, \& Marchant, 1996). Inulinase is produced by K. marxianus under aerobic conditions while its fermentation occurs under anaerobic conditions or with limited oxygen supply (Yuan, Zhao, Ge, \& Bai, 2008). So we put the hypothesis that ethanol was released into our yeast extracts. Moreover, the NADPHdependent alpha-keto amide reductase found in gel is in favor of the conversion of various carbonyl compounds into their corresponding alcohol products and this enzyme was studied in Saccharomyces cerevisiae (Chang, Griest, Harter, \& Petrash, 2007). The xylose fermentation to ethanol under anaerobic conditions 
(Zhang et al., 2015) can also be evoked in our study, since NAD(P)Hdependent D-xylose reductase or Xyl1 was found in gel for our strain $K$. marxianus S-02-5. This enzyme catalyzes the first step of the xylose fermentation. Thus, an ethanol release into the yeast extract could be expected in this study. To summarize, under anaerobic conditions, K. marxianus S-02-5 was likely limited in its growth and developed a fermentative metabolism at least partially using pentose pathway. Was this yeast subject to stress? Peptidylprolyl cis-trans isomerase was identified in the extract, which is known as a chaperone refolding aggregated proteins (Göthel \& Marahiel, 1999).

Both K. marxianus S-02-5 and K. marxianus MUCL 29917 demonstrated remarkable stability during their anaerobic incubation and in the presence of the mild detergent derived from cholic acid supposed to mimic intestinal conditions during the extraction process. Extracts prepared from these strains demonstrated strong action against $\mathrm{O}_{2}^{-}$as well as against $\mathrm{H}_{2} \mathrm{O}_{2}$. Moreover, these microorganisms were able to secrete antioxidant compounds at a higher level than did B. thetaiotaomicron ATCC 29741, previously studied for its antioxidant properties (Hochart-Behra et al., 2008, 2014). These compounds showed remarkable activity in vitro against superoxide anion and hydrogen peroxide, which are the first ROS described in the oxidative cascade of phagocytes (Hochart-Behra et al., 2008, 2014). Remarkably, these species were shown to be the first ROS produced in case of inflammatory burst by phagocytes, especially by neutrophils (Van Eeden, Klut, ..., \& Hogg, 1999).

K. marxianus species could be a good yeast candidate either to modulate or stop the oxidative-inflammatory cascade. Furthermore, K. marxianus was reported to exhibit stronger properties relative to the enhancement of transepithelial electrical resistance (TER) across a monolayer of differentiated human colonocytes (Caco-2 cells) (50\% TER increase) than probiotic S. boulardii $(30 \%$ TER increase) (Smith, Baker, Arneborg, \& Jespersen, 2015). K. marxianus, as well as Metschnikowia gruessii, were reported as the non-Saccharomyces yeasts to protect human epithelial cells from pathogen invasion (Smith et al., 2015), and the antioxidative effects evidenced here may explain, partially, the protective action of the $K$. marxianus species.

However, when K. marxianus S-02-5 and K. marxianus MUCL 29917 were cultured under aerobic atmosphere, their extracts did not show the same antioxidative potency. The protein contents of the extracts were also different depending on the mode of yeast incubation. Under anaerobic conditions, K. marxianus S-02-5 was more efficient. One hypothesis could be advanced that the alcoholic fermentation process could be at the origin of this difference. Indeed ethanol which is often used as solvent in the extraction of active natural products has been shown to have strong scavenger properties against ROS and probably to interfere with the effects that the authors seek to demonstrate. One should be vigilant before to attribute antioxidant effects to ingredients that are extracted with alcoholic solutions (Li, 2013). In our study, antioxidant proteins were not identified in the intense spots in gels for K. marxianus. Antioxidant compounds could be of lower molecular weight than $10 \mathrm{kDa}$, because the extracts were desalted on filters with cut-off level of $10 \mathrm{kDa}$. Some thiols of low molecular weight could be powerful. So, assays were finally performed on the corresponding filtrates that were stored in freezing to search their possible antioxidant power. Antioxidant effects were found with notable variations from one filtrate to the other which could be explained by the lability of ethanol or thiols that can be rapidly oxidized in disulphide bonds over time.

The intestinal content is characterized by anaerobic conditions, detergent medium and a strong representation of Bacteroides genus in intestinal flora. Basically we can expect the inhibition of the oxidative-inflammatory cascade in the presence of $K$. marxianus S-
02-5, especially in the case of inflammatory bowel diseases.

\section{Conclusion}

K. marxianus S-02-5 appears to bring a benefit for antioxidant activity when it is co-incubated with $B$. thetaiotaomicron under conditions mimicking the intestinal environment. Indeed, the antioxidant power of the extract performed from both microorganisms grown together was much more powerful than that obtained with the extract from the single bacterium. Moreover, K. marxianus S-02-5 exhibited strong inhibition of superoxide anion and hydrogen peroxide as well as the reference strain $K$. marxianus MUCL 29917. However, that could be due to ethanol fermentation. Animal models of inflammation will be of great interest in the study of the antioxidant and anti-inflammatory status of the tissues in contact with $K$. marxianus S-02-5. The data obtained in this study underpinned the antioxidative properties of $K$. marxianus species, mainly those of $K$. marxianus S-02-5, recently isolated from cheese, when it is incubated under anaerobic conditions in the presence of the $B$. thetaiotaomicron intestine symbiont. This attribute could then support the health claim of this yeast as a potential probiotic to counter intestinal inflammatory processes.

\section{Conflict of interest}

The authors do not declare any conflict of interest.

\section{Acknowledgements}

This work was realised at Charles Viollette Institute in University of Lille (France). Alexandre Ceugniez obtained a PhD scholarship from Ministère de l'Enseignement Supérieur et de la Recherche (France). The authors thank Portia Winters for her copyedited version of the manuscript. The REALCAT platform is benefiting from a governmental subvention administrated by the French National Research Agency (ANR) within the frame of the "Future Investments" program (PIA), with the contractual reference "ANR-11EQPX-0037". The Hauts-de-France region, the FEDER, Ecole Centrale de Lille and Centrale Initiative Foundation are warmly acknowledged for their financial contribution to the acquisition of the equipment of the REALCAT platform.

\section{Appendix A. Supplementary data}

Supplementary data related to this article can be found at http:// dx.doi.org/10.1016/j.lwt.2017.03.056.

\section{References}

Aruoma, O. I., Halliwell, B., Hoey, B. M., \& Butler, J. (1989). The antioxidant action of $\mathrm{N}$-acetylcysteine: Its reaction with hydrogen peroxide, hydroxyl radical, superoxide, and hypochlorous acid. Free Radical Biology and Medicine, 6(6), 593-597.

Bakolitsa, C., Xu, Q., Rife, C. L., Abdubek, P., Astakhova, T., \& Axelrod, H. L. (2010). Structure of BT_3984, a member of the SusD/RagB family of nutrient-binding molecules. Acta Crystallographica. Section F, Structural Biology and Crystallization Communications, 66, 1274-1280. http://dx.doi.org/10.1107/ S1744309110032999.

Banat, I. M., Singh, D., \& Marchant, R. (1996). The use of a thermotolerant fermentative Kluyveromyces marxianus IMB3 yeast strain for ethanol production (PDF Download Available). Acta Biotechnology, 16(2-3), 215-223. http:// dx.doi.org/10.1002/abio.370160223.

Cammarota, G., Ianiroa, G., Cianci, R., Bibbò, S., Gasbarrini, A., \& Currò, D. (2015). The involvement of gut microbiota in inflammatory bowel disease pathogenesis: Potential for therapy. Pharmacology \& Therapeutics, 149, 191-212. http:// dx.doi.org/10.1016/j.pharmthera.2014.12.006.

Cardenas, J. P., Quatrini, R., \& Holmes, D. S. (2016). Aerobic lineage of the oxidative stress response protein rubrerythrin emerged in an ancient microaerobic, (Hyper)Thermophilic environment. Frontiers in Microbiology, 7, 1822. http:// dx.doi.org/10.3389/fmicb.2016.01822. 
Ceugniez, A., Drider, D., Jacques, P., \& Coucheney, F. (2015). Yeast diversity in a traditional French cheese "Tomme d'orchies" reveals infrequent and frequent species with associated benefits. Food Microbiology, 52, 177-184. http:// dx.doi.org/10.1016/j.fm.2015.08.001.

Chang, Q., Griest, T. A., Harter, T. M., \& Petrash, J. M. (2007). Functional studies of aldo-keto reductases in Saccharomyces cerevisiae. Biochimica et biophysica acta, 1773, 321-329. http://dx.doi.org/10.1016/j.bbamcr.2006.10.009.

De Biase, D., Tramonti, A., Bossa, F., \& Visca, P. (1999). The response to stationaryphase stress conditions in Escherichia coli: Role and regulation of the glutamic acid decarboxylase system. Molecular Microbiology, 32(6), 1198-1211. mmi1430 [pii].

Dellomonaco, C., Amaretti, A., Zanoni, S., Pompei, A., Matteuzzi, D., \& Rossi, M. (2007). Fermentative production of superoxide dismutase with Kluyveromyces marxianus. Journal of Industrial Microbiology \& Biotechnology, 34, 27-34. http:// dx.doi.org/10.1007/s10295-006-0158-4.

Fischbach, Michael A., \& Sonnenburg, Justin L. (2011). Eating for Two: How metabolism establishes interspecies interactions in the gut. Cell Host \& Microbe, 10 , 336-347. http://dx.doi.org/10.1016/j.chom.2011.10.002.

Fujimoto, M., Yamada, A., Kurosawa, J., Kawata, A., Beppu, T., \& Takano, H. (2012). Pleiotropic role of the Sco1/SenC family copper chaperone in the physiology of streptomyces. Microbial Biotechnology, 5, 477-488. http://dx.doi.org/10.1111/ j.1751-7915.2011.00319.x.

Göthel, S. F., \& Marahiel, M. (1999). Peptidyl-prolyl cis-trans isomerases, a superfamily of ubiquitous folding catalysts. Cellular and Molecular Life Sciences, 65(3), 423-436. http://dx.doi.org/10.1007/s000180050299.

Hochart-Behra, A. C., Behra-Miellet, J., Sam, J., Drobecq, H., Gressier, B., \& Luyckx, M. (2008). Antioxidative effect of Bacteroides thetaiotaomicron extracts: Superoxide dismutase identification. Analytical and Bioanalytical Chemistry, 391(1), 415-423. http://dx.doi.org/10.1007/s00216-008-1993-0.

Hochart-Behra, A. C., Drobecq, H., Tourret, M., Dubreuil, L., \& Behra-Miellet, J. (2014). Anti-stress proteins produced by Bacteroides thetaiotaomicron after nutrient starvation. Anaerobe, 28, 18-23. http://dx.doi.org/10.1016/ j.anaerobe.2014.04.008.

Kelly, D., Campbell, J. I., King, T. P., Grant, G., Jansson, E. A., \& Coutts, A. G. (2004), Commensal anaerobic gut bacteria attenuate inflammation by regulating nuclear-cytoplasmic shuttling of PPAR-gamma and RelA. Nature Immunology, 5(1), 104-112. http://dx.doi.org/10.1038/ni1018.

Leavey, P. J., Gonzalez-Aller, C., Thurman, G., Kleinberg, M., Rinckel, L., \& Ambruso, D. W. (2002). A 29-kDa protein associated with p67phox expresses both peroxiredoxin and phospholipase A2 activity and enhances superoxide anion production by a cell-free system of NADPH oxidase activity. Journal of Biological Chemistry, 47(22), 45181-45187. http://dx.doi.org/10.1074/ jbc.M202869200.

Li, X. (2013). Solvent effects and improvements in the deoxyribose degradation assay for hydroxyl radical-scavenging. Food Chemistry, 141, 2083-2088. http:// dx.doi.org/10.1016/j.foodchem.2013.05.084.

Lowry, O. H., Rosebrough, N. J., Farr, A. L., \& Randall, R. J. (1951). Protein measurement with the Folin phenol reagent. Journal of Biological Chemistry, 193(1), $265-275$.

Luo, T., Fan, T., Liu, Y., Rothbart, M., Yu, J., \& Zhou, S. (2012). Thioredoxin redox regulates ATPase activity of magnesium chelatase CHLI subunit and modulates redox-mediated signaling in tetrapyrrole biosynthesis and homeostasis of reactive oxygen species in pea plants. Plant Physiology, 159, 118. http:// dx.doi.org/10.1104/pp.112.195446.

Maccaferri, S., Klinder, A., Brigidi, P., Cavina, P., \& Costabile, A. (2012). Potential probiotic Kluyveromyces marxianus B0399 modulates the immune response in Caco-2 cells and peripheral blood mononuclear cells and impacts the human gut microbiota in an in vitro colonic model system. Applied Environmental
Microbiology, 78(4), 956-964, 0.1128/AEM.06385-11.

Martynova, J., Kokina, A., Kibilds, J., Liepins, J., Scerbaka, R., \& Vigants, A. (2016) Effects of acetate on Kluyveromyces marxianus DSM 5422 growth and metabolism. Applied Microbiology and Biotechnology, 100, 4585-4594. http:// dx.doi.org/10.1007/s00253-016-7392-0.

Miller, T. L., \& Wolin, M. J. (1979). Fermentations by saccharolytic intestinal bacteria. The American Journal of Clinical Nutrition, 32, 164-172.

Mishra, S., \& Imlay, J. A. (2013). An anaerobic bacterium, Bacteroides thetaiotaomicron, uses a consortium of enzymes to scavenge hydrogen peroxide. Molecular Microbiology, 90, 1356-1371. http://dx.doi.org/10.1111/mmi.12438.

Nedeva, T., Dolashka-Angelova, P., Moshtanska, V., Voelter, W., Petrova, V., \& Kujumdzieva, A. (2009). Purification and partial characterization of $\mathrm{Cu} / \mathrm{Zn}$ superoxide dismutase from Kluyveromyces marxianus yeast. Journal of Chromatography. B, Analytical Technologies in the Biomedical and Life Sciences, 877, 3529-3536. http://dx.doi.org/10.1016/j.jchromb.2009.08.015.

Petrof, E. O., \& Khoruts, A. (2014). From stool transplants to next-generation microbiota therapeutics. Gastroenterology, 146, 1573-1582. http://dx.doi.org/ 10.1053/j.gastro.2014.01.004.

Pinheiro, R., Belo, I., \& Mota, M. (2002). Oxidative stress response of Kluyveromyces marxianus to hydrogen peroxide, paraquat and pressure. Applied Microbiology and Biotechnology, 58, 842-847. http://dx.doi.org/10.1007/s00253-001-0927-y.

Ridlon, J. M., Kang, D. J., \& Hylemon, P. B. (2006). Bile salt biotransformations by human intestinal bacteria. Journal of Lipid Research, 47(2), 241-259. R500013JLR200 [pii] 10. 1194/jlr.R500013-JLR200.

Rouwenhorst, R. J., Hensing, M., Verbakel, J., Scheffers, W. A., \& van Duken, J. P. (1990). Structure and properties of the extracellular inulinase of Kluyveromyces marxianus CBS 6556. Applied and Environmental Microbiology, 56, 3337-3345.

Shevchenko, A., Wilm, M., Vorm, O., \& Mann, M. (1996). Mass spectrometric sequencing of proteins silver-stained polyacrylamide gels. Analytical Chemistry 68(5), 850-858, 0003-2700/96/0368-0850\$12.00/0.

Singh, R. S., \& Lotey, S. (2010). Enhanced exoinulinase production from Kluyveromyces marxianus YS-1 using response surface methodology. Brazilian Archives of Biology and Technology, 53, 1005-1013. http://dx.doi.org/10.1590/S151689132010000500002.

Smith, I. M., Baker, A., Arneborg, N., \& Jespersen, L. (2015). Non-Saccharomyces yeasts protect against epithelial cell barrier disruption induced by Salmonella enterica subsp. enterica serovar Typhimurium. Letter in Applied Microbiology, 61(5), 491-497. http://dx.doi.org/10.1111/lam.12481.

Stefanova, T. H., Ratcheva, I., Nikolova, N. J., \& Neychev, H. O. (2014). Effect of yeast superoxide dismutase treatment on some mediators of inflammation during adjuvant-induced arthritis in mice. Zeitschrift für Naturforschung C, 65, 141-147. http://dx.doi.org/10.1515/znc-2010-1-222.

Van Eeden, S. F., Klut, M. E., \& Hogg, J. C. (1999). The use of flow cytometry to measure neutrophil function. Journal of Immunological Methods, 232(1), 23-43. http://dx.doi.org/10.1016/S0022-1759(99)00148-9.

$\mathrm{Xu}$, J., \& Gordon, J. I. (2003). Inaugural Article: Honor the symbiont. Proceeding of the National Academy of Science U S A, 100(18), 10452-10459. http://dx.doi.org/ 10.1073/pnas.17340631001734063100 [pii].

Yuan, W. j., Zhao, X. q., Ge, X. m., \& Bai, F. w. (2008). Ethanol fermentation with Kluyveromyces marxianus from Jerusalem artichoke grown in salina and irrigated with a mixture of seawater and freshwater. Journal of Applied Microbiology, 105, 2076-2083. http://dx.doi.org/10.1111/j.1365-2672.2008.03903.x.

Zhang, B., Zhang, J., Wang, D., Gao, X., Sun, L., \& Hong, J. (2015). Data for rapid ethanol production at elevated temperatures by engineered thermotolerant Kluyveromyces marxianus via the $\mathrm{NADP}(\mathrm{H})$-preferring xylose reductase-xylito dehydrogenase pathway. Data in Brief, 5, 179-186. http://dx.doi.org/10.1016/ j.dib.2015.08.038. 